\title{
Das Deutsch der Migranten - eine Zwischenbilanz (Podiumsdiskussion auf der 48. Jahrestagung des Instituts für Deutsche Sprache)
}

\section{Die Einführung (durch den Moderator, Ludwig M. Eichinger)}

Auf der Podiumsdiskussion, die am Ende des zweiten Tagungstages angesetzt war, ging es einerseits darum, Dinge zu sortieren, die im bisherigen Tagungsverlauf schon eine Rolle gespielt hatten. Von den Personen, die auf dem Podium saßen, war außerdem zu erwarten, dass sie nicht nur von ihren jeweiligen Tätigkeitsprofilen her Neues zur thematischen Diskussion beizutragen hätten, sondern auch von ganz unterschiedlichen Richtungen an unser Thema herangehen würden.

Wenn man betrachtet, worauf bei der Podiumsdiskussion bereits zurückgeblickt werden konnte, so waren das die Referate des ersten Tages, in denen verschiedene linguistische und soziolinguistische Deutungsmöglichkeiten der durch das Tagungsthema angedeuteten sprachlichen und gesellschaftlichen Lagen präsentiert worden waren, und die Beiträge des zweiten Tages, in denen es um einschlägige Sprachbiographien, individuellere Muster des Sprachgebrauchs in mehrsprachigen Kontexten und deren theoretische Modellierung ging.

Auf dem Podium saßen die drei Linguisten Jannis Androutsopoulos (Hamburg), Konrad Ehlich (Berlin) und Rita Franceschini (Bozen), dazu kam Matthias Makowski (München), der Leiter der Sprachabteilung des Goethe-Instituts. In der Reihenfolge der Vorstellung kam als Erste Frau Franceschini, die den Lehrstuhl für allgemeine Sprachwissenschaft und Soziolinguistik an der Universität Bozen innehat. Gleichzeitig ist sie dort Vorsitzende des „Zentrums für Mehrsprachigkeit", einer Institution, die im Kontext der mehrsprachigen Landschaft von Südtirol in Bozen eine Art natürlichen Sitz hat - auf jeden Fall, wenn man das vom deutschen Sprachgebiet aus sieht, in dem die Südtiroler Verhältnisse einen paradigmatischen Mehrsprachigkeitsfall darstellen. Sie ist aber nicht erst dort zur Mehrsprachigkeitsforscherin geworden, sondern hat ihre Erfahrungen, die sie nicht zuletzt an den Schweizer Verhältnissen gemacht hat, dorthin mitgebracht. Sie bringt dadurch verschiedenartige Erfahrungen in die Diskussion ein. So hat sie sich einerseits mit den neuronalen Verhältnissen beim sprachlichen Agieren mehrsprachiger Sprecherinnen und Sprecher auseinandergesetzt, sie hat aber auch erst unlängst als neue Herausgeberin der Zeitschrift für Literaturwissenschaft und Linguistik ein Themenheft zu Sprachbiographien 
erscheinen lassen. Ein zweites Statement wurde von Konrad Ehlich vorgetragen, der von 1992 bis zu seiner Emeritierung im Jahr 2007 Leiter des lnstituts für Deutsch als Fremdsprache/Transnationale Germanistik an der Universität München war und nun seit 2009 Honorarprofessor an der Freien Universität Berlin ist. Auch hier seien von den vielen Anknüpfungspunkten, die seine wissenschaftliche Tätigkeit für das Thema des Podiums böte, nur zwei genannt: seine langjährige Tätigkeit als führender Forscher im Bereich des Deutschen als Fremd- und als Zweitsprache, und in der theoretischen Modellierung dieser Bereiche kommt ihm in diesem Umfeld eine herausgehobene Rolle zu. Dazu kommt aber, dass er das Phänomen Mehrsprachigkeit aus einander ergänzenden Perspektiven fokussiert: zum einen als Theoretiker einer Pragmatik der Mehrsprachigkeit, dazu aber auch unter gesellschaftspolitischem Aspekt als der Sprachsoziologe, als der er sich ebenfalls vielfach geäußert hat. Als ehemaliger Vorsitzender des Germanistenverbandes stellt er in unserem Kreis auch eine Art offizielle germanistische Instanz dar. Der dritte Linguist in der Runde war Jannis Androutsopoulos, Professor für Linguistik des Deutschen/Medienlinguistik an der Universität Hamburg und Fellow am FRIAS (Freiburg Institute for Advanced Studies). Er ist einer der Linguisten, die sich am intensivsten mit Jugend- und Mediensprache auseinandergesetzt haben. Verschiedene seiner Tätigkeitsfelder sind für unser Podium einschlägig, so hat er ebenso intensiv zur Heteroglossie in der Populärkultur geforscht wie er Ethnolekte und urbane Zwei- und Mehrsprachigkeit unter Jugendlichen wissenschaftlich in den Blick genommen hat. Von der „Erfindung der Ethnolekte“ hat er unlängst geschrieben, und das sollte auch hier Anlass zur Diskussion bieten.

Abgerundet wurde das Podium durch Matthias Makowski, den Leiter der Spracharbeit des Goethe-Instituts. Er ist auch Germanist, und hat bei einem der Altpräsidenten des IDS, Siegfried Grosse, promoviert, wichtiger für uns ist aber sicherlich, dass wir mit ihm in seiner Funktion im GoetheInstitut eine Person auf dem Podium hatten, die sich praktisch mit Fragen der sprachlichen Integration, ihren gesetzlichen Umständen, ihrer didaktischen Praxis und ihrer Handhabung in einem demokratischen Sinne zu beschäftigen hat. Er ist amtlich verantwortlich für den Bereich Integrationskurse, Sprachkurse für Zuzügler, Sprachprüfungen dieses Typs und ähnliche Dinge. Dadurch hat er Erfahrungen und Kompetenzen, die bei solch einer Diskussion nicht fehlen dürfen. 


\section{Die Eingangsbeiträge des Podiums ${ }^{1}$}

[Moderation] Jannis Androutsopoulos versprach mit den angedeuteten Thesen zur „Erfindung“ der Ethnolekte sich gegenüber dem Tagungsthema, so wie es formuliert war, am kritischsten zu positionieren, damit sollte er das Podium eröffnen.

\subsection{Jannis Androutsopoulos (Hamburg)}

Ich will im Anschluss an meine im Vorfeld dieser Veranstaltung angestellten Überlegungen, aber auch aufgrund der Eindrücke und Ideen, die sich während der Tagung herauskristallisiert haben, vier Punkte in aller Kürze und daher auch einigermaßen pointiert festhalten. Ausgehen will ich dabei von der grundlegenden Feststellung, dass jegliche Vorstellung von Sprache und von Sprachen generell diskursiv hergestellt wird. Solche Vorstellungen entstehen also in einer metasprachlichen Auseinandersetzung reflexiver Praktiken, wie das auch im Laufe dieser Tagung schon formuliert wurde. Auf diesem Weg entstehen gemeinsam geteilte Vorstellungen von Sprache und Dialekten und von sprachlichen Kontakten. Im Grundsatz gilt das auch für die Vorstellung vom „Deutsch der Migranten“. Das Überraschende ist aber vielleicht, dass das „Deutsch der Migranten“ in der diskursiven Aushandlung der Nicht-Migranten gemacht wird. Dass diese diskursive Konstruktion hauptsächlich von Nicht-Migranten hergestellt wird, gilt mehr oder weniger für die Sprachwissenschaft, das gilt aber auch für andere Wissenschaften, das gilt vermutlich umso mehr für die Sprachpolitik, also für verschiedene Modellierungen des Zusammenhangs von Sprache und Integration auf bundes- wie auf landespolitischer Ebene.

Was ich mir an dieser Stelle wünschen würde, wäre ein stärkerer Einbezug der Innenperspektive, mehr Beteiligung von Menschen mit Migrations- und Mehrsprachigkeitserfahrung an diesen diskursiven Herstellungsprozessen. Um nicht missverstanden zu werden: Damit soll nicht den Nicht-Migranten das Einfühlungsvermögen in diese Fragestellung abgesprochen, noch soll damit der migrantische Hintergrund zum Allheilmittel für eine erfolgreiche Forschung in diesem Bereich erklärt werden. Gesagt werden soll nur: Mehr Innenperspektiven wären interessant, wären fruchtbar, würden auch andere Fragestellungen als die jetzt üblichen in den Vordergrund bringen.

Damit zu den angekündigten vier Punkten. Der erste Punkt ist: Zwar wird in der wissenschaftlichen Diskussion - auch auf dieser Tagung versucht, an das „Deutsch der Migranten“ möglichst unvoreingenommen

Die Beiträge werden hier größtenteils jeweils in den eigenen Worten der Teilnehmer der Podiumsdiskussion wiedergegeben. 
heranzugehen, ja die Existenz eines einheitlichen Phänomens mit diesem Namen infrage zu stellen. Dennoch ist nach wie vor die Tendenz in unserer Diskussion unübersehbar, dass es bei dem, was wir tatsächlich erforschen, dann doch noch ziemlich viel nur um die Benachteiligten, Unterprivilegierten, die Menschen mit geringem gesellschaftlichem Kapital, Menschen, denen geholfen werden muss, geht. Das ist natürlich ein notwendiger Blickwinkel: Mir scheint aber darin dennoch eine gewisse Verengung der Bandbreite von Migrationstypen und -karrieren und auch der Bandbreite von sprachlichen Kompetenzen, die damit einhergehen, stattzufinden. Wo sind - und auch das wurde während der Tagung schon einmal angemahnt - wo sind dann die migrantischen Regisseure, Fußballspieler, Unternehmer, Politiker und so weiter? Also nicht die Personen, die aus nicht-bildungsfernen Schichten kommen, die aber dennoch einen migrantischen Hintergrund haben, sich dann eben das Deutsche als Fremd- oder Zweitsprache angeeignet haben und dann auch so verwenden. Für einen angemessenen Blick braucht es eine Erweiterung des Spektrums jenseits der Benachteiligungsperspektive, die sich - wenn auch sicher nicht absichtlich in den Vordergrund gerückt - immer noch in der Auswahl unserer Forschungsgegenstände niederschlägt.

Der zweite Punkt: Dass das Deutsch der Migranten, so die These, hauptsächlich von Nicht-Migranten diskursiv hergestellt wird, führt in der sprachwissenschaftlichen Betrachtung und vor allem dann auf einer sprachpolitischen Ebene zu einer Loslösung des Deutschen von komplexeren mehrsprachigen Praktiken. Dagegen ist der Gebrauch des Deutschen aus der Perspektive von Mehrsprachigen, von Migranten, immer Folge eines komplexen Spracherwerbs, und es ist Teile einer komplexen sprachlichen Praxis, wo immer es verwendet wird, sei es im Alltag, im Beruf, in der Profession oder wo sonst. Was bringt da diese Loslösung, diese Herauslösung des Deutschen? Sie ist weder realistisch noch sprachpolitisch zielführend. Wie sehr kann man letzten Endes gute Kampagnen für das Deutsche machen, wenn man die Mehrsprachigkeit im Leben von Migranten nicht symbolisch anerkennt. Dass das geschieht, kann man an verschiedenen Kampagnen für Sprache und Integration sehen, wenn sie mit Schlagzeilen wie den folgenden für den Gebrauch des Deutschen werben: „Sag's auf Deutsch!“ oder „Trau Dich! Sag's auf Deutsch“. Verstärkt wird dieses monolinguale Konzept durch Clips mit den Brüdern Klitschko und anderen Prominenten, die alle stolz verkünden: „Ich habe mich auch getraut. Ich habe es auch auf Deutsch gesagt.“ Diese ganzen Kampagnen geben keine Hinweise auf die vorhandene Mehrsprachigkeit, vielmehr marginalisieren sie die Mehrsprachigkeit symbolisch. Sie befinden sich damit im Fahrwasser einer Konzeption der doppelten Einsprachigkeit. Mit solchen Kanpagnen kommuniziert uns die Bundesregierung: „Es ist wichtig, dass Du in diesem Land Deutsch lernst.“ Dass dieses Deutsch in der Realität immer Teil mehr- 
sprachiger Praktiken ist und dass das für alle Beteiligten von Vorteil ist, wird aber eben nicht erwähnt und damit auch nicht symbolisch gewürdigt. Es wäre sinnvoll, mehr in diese Richtung zu gehen, und dass das geht, sieht man an Werbekampagnen, die von der Wirtschaft lanciert werden, und in denen Zweisprachigkeit oder genereller Mehrsprachigkeit zu - positiv bewerteten - Identitätsmerkmalen von beworbenen Kunden stilisiert werden.

Der dritte Punkt: Das „Deutsch der Migranten“ wird nach wie vor ziemlich stark unter den Vorzeichen von „Standardismus“ und „Homogenismus“ diskursiv bearbeitet. Standardismus meint das Postulat des Vorrangs der Standardsprache, das quasi sprachideologisch zur Messlatte und zum Standard für alles andere deklariert wird. Homogenismus meint das Postulat der Einheitlichkeit. Das Postulat der Einheitlichkeit von Varietäten einschließlich der Standardsprache. Man erkennt, wie diese beiden Postulate implizit wirken, insbesondere an der Art und Weise, wie Ethnolekte und Ähnliches in den Medien verhandelt werden. Dort werden sprachliche Praktiken, die doch sehr flüssig sind und sehr veränderlich und anpassungsfähig, plötzlich einfach zu fest umgrenzten Idiomen, Stilen mit Begrenzungen. Sie tragen dann auch Namen, oder: Etiketten, mit denen bestimmte Merkmale - oder deren Fehlen - assoziiert werden. Hier wird vor allem in einer Medienpraxis, aber durchaus auch in der Sprachwissenschaft eine eigene und spezifische Homogenität erschaffen. Ich will gerne zugeben, dass ich hier auch aus Fehlern der eigenen Vergangenheit gelernt habe. Worum es in der Sache geht, wird klar, wenn man sich ansieht, wie diese Benennungen und Etiketten gestaltet sind. Sie heißen „Kanakisch“, „Kanaksprach“, „Ethnolekt" oder „Migrantenslang“. Diese ganzen Labels sind nicht einfach nur deskriptive Statements, sie rufen vielmehr weitere - bewertende diskursive Prozesse auf.

Vierter und letzter Punkt: Es ist sicher einiges wahr an der unter Linguisten verbreiteten Feststellung, dass die Linguisten in der öffentlichen Diskussion generell kein Gehör fänden. Dennoch ist aber genauso wahr, dass ihre Statements doch dort eingesetzt werden; die linguistischen Kompetenzen, wenn auch im konkreten vielleicht nicht so sehr geschätzt, sind als Stützen von Diskurspositionen unverzichtbar in der ganzen Berichtserstattung über Varietäten des Deutschen, Sprache und Integration und ähnliche Themen. Die Position, dass das eine die Fiktion der Medien sei und auf einem ganz andern Blatt die Wirklichkeit der Sprachwissenschaftler stehe, ist zu einfach. Auch Linguisten sind, sobald sie sich öffentlich äußern, Teil dieser Fiktionen, nicht zuletzt durch die Präkontextualisierung wissenschaftlicher Statements in der medialen Berichterstattung. Die Verschränkung zwischen den beiden Welten einzubeziehen und ernst zu nehmen, wäre ein wichtiger Schritt im Sinne der wissenschaftlichen „Korrektur“ der öffentlichen Diskurse - nicht nur über Sprache und Migration. 
[Moderation]: Weil ihr Beitrag in gewisser Weise an den ersten anschließt, weil es in ihm auf jeden Fall auch um die Notwendigkeit einer differenzierten Betrachtung, vielleicht sogar um eine notwendige Individualisierung geht, ist der nächste Beitrag der von Rita Franceschini.

\subsection{Rita Franceschini (Bozen)}

Die Kunst auf einem solchen Podium, auf dem sich die Teilnehmer, wenn sie das Thema ernsthaft besprechen, oft gar nicht so uneinig sind, liegt darin, dann doch - durchaus im Sinne der Wahrheitsfindung, aber nicht zuletzt auch zur Unterhaltung des Publikums - gegensätzliche Positionen zu inszenieren. Deshalb versuchen wir auch ein bisschen pointiert zu sagen: „Wir können es aber auch anders offerieren.“ Deshalb möchte ich mit einer Reaktion beginnen, die ich hatte, als ich die Einladung bekommen habe und natürlich wissend, was das IDS ist und mit allem Respekt gegenüber allen Personen, die ich aus dem IDS kenne, dachte: „Das ist schon ein komischer Titel!“", der eigentlich sagt: Es ist Deutsch, das Deutsch der Migranten. Aber nicht nur das. Stellen Sie sich vor, man hätte eine Tagung, die heißt: „Das Deutsch der Deutschen. " Dann wären Sie in der Lage, Sie müssten festlegen, was die Deutschen sind. Hoppala! Also das wäre eine ganz brisante Frage. Das würden Sie nie tun. Oder?

Dann hätte man dieselbe Definitionssituation wie jetzt, wo man beim Titel dieser Tagung ja sagen müsste, was sind eigentlich Migranten, was ist das Deutsch. Wenn wir das tun wollten, kämen wir wie alle anderen in eine riesige Bredouille, aber - eine provokante These - man traute sich das doch eher gegenüber Personengruppen, die man noch als randständig betrachtet. Da hat man die Definitionsmacht, da übt man sie aus, da guckt man sich diese verschiedenen Gruppen und Personen in ihren Eigenheiten an. Dabei sieht man, dass man, wenn man die sprachlichen Auffälligkeiten, die man beobachtet, nur als Fehler sieht, in die Irre geführt wird. Wenn man genauer hinsieht, bemerkt man, dass eben beispielsweise bei Spracherwerb, auch beim Mehrspracherwerb, ähnliche Phänomene auftreten, nicht immer die gleichen, nicht in derselben Häufigkeit, auch doch ähnliche Phänomene: Kasus, Genus etc. sind noch nicht fix. Deshalb auch meine These, dass zwar das Deutsch der Migranten, wenn es das in dieser Allgemeinheit gibt, oder seine Sprecher zum Deutschen ein anderes Verhältnis haben als die monolingualen Sprecher der Mehrheitssprache. Im Vergleich zu ihnen können sie als Randgruppen verstanden werden, allerdings als Randgruppen, die unter den heutigen Verhältnissen in Europa ebenso einen gleichberechtigten Zugang zum Deutschen haben. Diese Personen haben andere Lebenswege, was das Sprachenlernen angeht, und daher können sie das Deutsche anders aussprechen und anders nutzen. Wenn man als Linguist allerdings 
genau betrachtet, in welchen Bereichen der Grammatik eigentlich bei einem solchen Typ von Spracherwerb Schwankungen vorkommen, sieht man, dass sowohl beim Erstspracherwerb, als auch beim Zweitspracherwerb, insgesamt die Merkmale dieses speziellen Deutsch erscheinen, das eben nicht das „fertige“ Deutsch von monolingualen Sprechern ist. Man zeige mir im Übrigen jemanden, der immer nur monolingual aufwächst und lebt. Obwohl wir uns fragen müssen, ob es das überhaupt gibt, nehmen wir das als Norm an. Die Sprecher, die nicht einen solchen Zugang haben, lernen Deutsch jedenfalls mit charakteristischen Merkmalen derjenigen Anteile im Deutschen, die generell nicht so fest fixiert sind. Sie legen uns also damit eigentlich dar, welches die schwachen Stellen im grammatischen System sind. Diese beobachteten Auffälligkeiten an diesen fluktuierenden Stellen des Sprachsystems sind Indikatoren, eine Art Lackmuspapier, um die Festigkeit des Sprachsystems zu testen.

Aber auch in anderer Hinsicht möchte ich das einheitliche und klassifizierende Reden vom „Deutsch der Migranten“ in Frage stellen. Ich denke, es gibt noch eine andere Sicht auf die ganze Sache, auf die ich eigentlich hinwirken möchte. In ihrer vermeintlichen Neutralität ist diese Formulierung eigentlich fast eine Art Euphemismus. Ruft sie doch in der prototypischen Sicht die Vorstellung von Unterschichtsmigranten, Migranten der zweiten und vielleicht der dritten Generation wach. Andere Vertreter kommen eigentlich nicht in den Fokus, die man sehen könnte, wenn man den Blick weiten würde, nämlich Personen, die mit einem mehrsprachigen Hintergrund eben in ihrem Repertoire auch Deutsch haben. Die Wendung zu dieser Vielfalt von sprachlichen Verhältnissen ist die neue Sicht, die auf dieser Tagung bei den Beiträgen zu den Sprachbiographien ganz deutlich geworden ist. Das hier von den meisten benutzte Konzept des Repertoires entspricht auf der personellen Ebene dem Begriff des flexiblen Sprechers. Eines Sprechers also, der sich in seinem Repertoire einer ganzen Bandbreite von sprachlichen Optionen bedienen kann, und sich mittels dieses Stils einer adaptiven Vielfalt, mit diesen ganzen verschiedenen Repertoires und Kombinationen davon eine soziale Identität gibt, die von anderen so gesehen wird, durch die er sich aber eben auch selbst definiert. Eine solche Ausprägung sozialer Identität finden wir nicht zuletzt bei Jugendlichen, die mit der Vielfalt ihrer Sprachen und Sprechweisen, mit diesem Repertoire jonglieren. Man spricht von der Bricolage, einem Basteln mit Sprachen. Da werden Dinge neu zusammengesetzt und man mokiert sich auf diese Weise manchmal auch über das an Homogenität und Monolingualität orientierte Kategorisieren der sprachlichen Welt. Die individuelle Sicht auf die Konstruktion der Wirklichkeit in einer Person, die mit der Analyse von Sprachbiographien geleistet wird, ergänzt so die zweifellos nötige Frage nach den Bedingungen des sozialen Kontextes. Man muss das nicht verabsolutieren, aber man sieht bei dieser Sicht auf das Individuum mit seinen Möglich- 
keiten den ganzen Reichtum und auch die Veränderbarkeit des eigenen Repertoires. Man sieht auch, dass Personen, die mit einer Sprache, oder zwei, aufgewachsen sind, die eine z.B. im Alter von dreißig Jahren besser sprechen, um mit fünfzig die Dominanzverhältnisse zu wechseln. Es gibt auch Verläufe, die so reichhaltig sind, dass wir ihrer mit unseren sprachwissenschaftlichen Mitteln noch nicht so ganz Herr geworden sind.

Die dritte These wäre: Was wir vor allem bei den Jugendlichen gesehen haben, ist eigentlich „Neudeutsch“, eine generell neue Art von Deutsch. Auch Jugendliche, die keinen Migrationshintergrund haben, sondern eher in monolingualen Kontexten aufgewachsen sind, reden Deutsch nicht wie die vorhergehende Generation. Sie nutzen andere sprachliche Mittel. Nicht zuletzt stehen sie im Kontakt mit Mitgliedern ihrer Alterskohorte, die eine andere sprachliche Sozialisationsgeschichte haben, und von denen sie viele sprachliche Mittel mitbekommen. Sie reichern ihr Repertoire an, oft auch nur mit Versatzstücken, also einzelnen Konstruktionen, die sie dann in ihr eigenes Deutsch einbauen. Dieser Prozess des Einbauens in die eigene Rede ist wesentlich. Die Soziolinguistik hat derzeit noch nicht die Mittel, um diese Art von Variabilität, diese viel höhere Variabilität, zu erfassen. Hier bedarf es einer Methodendiskussion, vielleicht nicht einmal mit dem Ziel, die ganze Bandbreite von Mehrsprachigkeit in den Griff zu bekommen; wichtig wäre es zunächst einmal, diesen sprachlichen Reichtum einfach einmal in adäquater Weise darstellen zu können. Eine adäquate Modellierung wäre eine, die auch die Akteure für sich akzeptieren könnten. So gesehen ist die Titelformulierung dieser Tagung schon in Ordnung, allerdings muss einem klar sein, dass sie eine Kategorisierung von außen darstellt und daher nur für eine bestimmte Bandbreite von Erscheinungen adäquat ist.

[Moderation]: Ich gebe ohne viele Worte an Herrn Ehlich weiter, von dem wir uns erhofft haben, dass er die deutschen Verhältnisse, deren Komplexität sich die bisherigen Diskutanten gewidmet haben, im Hinblick auf eine transnationale Perspektive einer europäischen Mehrsprachigkeit öffnet.

\subsection{Konrad Ehlich (Berlin)}

Ich will zu fünf Punkten kurz etwas bemerken.

Der erste betrifft ebenfalls dieses Konstrukt der Linguistik, das sich im Titel dieser Tagung findet: „Das Deutsch der Migranten“. Schon im ersten Vortrag wurde der allgemeine Anspruch dieser Formulierung reduziert: Eine ganze Reihe von Migranten wurde von vorneherein gleich einmal außer Betracht genommen. Eigentlich ist aber die Realität unserer sprachlichen Situation in Deutschland und in Europa insgesamt durch eine Vielfalt auch der Typen von Migration gekennzeichnet, was durch diese einheitliche und 
generalisierende Formulierung eher verdeckt wird. Wir haben mit einem breiten Spektrum von Varietäten zu rechnen, die sich in unseren Gesellschaften finden lassen. Sie sind zweifellos von größerer und geringerer Permanenz. Wir haben Anlass, das bei unserem linguistischen Tun nicht auszublenden, sondern als Objekt unserer Beschreibung und Analyse ernst zu nehmen. Die Realität dieser verschiedenen Varietäten macht sich nicht zuletzt im Sprachwissen der Sprechenden bemerkbar. Wie sehr wir dort differenzieren können, mag sich je nach dem Objekt der Untersuchung und den sozialen Bedingungen des Sprachgebrauchs unterscheiden, ob es z.B. um die Klassifikation neuer Phänomene wie „Kiezdeutsch“ oder historische Stufen der Variation (z.B. „Obersächsisch“) geht. Wir haben eine Realität des sprachlichen Individuums und so neu ist auch das eigentlich nicht. Wir haben in gewisser Weise analoge Prozesse mit der polnischen Migration gegen Ende des 19. Jahrhunderts und der Einschätzung dessen, was daraus entstanden ist (z.B. Bewertung von „Ruhrgebietsdeutsch"), und hierher gehört auch die „mehrsprachige“ Realität dessen, was mit der Dichotomisierung in die Kategorien Dialekt und (Hoch-)Sprache aus dieser eigentlich angemessenen systematischen Sichtweise auch so ein bisschen aus dem Blick herausgenommen worden ist.

Zweiter Punkt: Das mehrsprachige Individuum in Europa lebt in einer Situation der konsolidierten Einsprachigkeit. Sie ist das Ergebnis des Projekts Nation, eines offenbar unheimlich erfolgreichen Projekts, das von Europa ausgehend inzwischen praktisch die ganze Welt erreicht hat, mit allen Positiva und allen Negativa. Wenn wir auf die Positiva schauen, dann ist die Standardvarietät, ist die Hochsprache, zunächst einmal nur eine bedeutende sowohl horizontal wie vertikal wirksame große Ressource für Kommunikation in großen Räumen. Horizontal eben diatopisch, vertikal als schichtenübergreifend und insofern als etwas, was in diesen sehr komplexen Gesellschaften eine zentrale Rolle spielt. Zu den Negativa gehört, dass in Folge dieses Konzepts das Bild des einsprachigen Individuums das Leitbild und das gesellschaftliche Ideal darstellte - eine Sicht, die durchaus auch der Linguistik nicht fremd war. Es ist die Folge großer historischer Veränderungsprozesse, dass sich ein Denkmodell herausgebildet hat, bei dem Mehrsprachigkeit neben Einsprachigkeit steht. Das ist sowohl gesellschaftlich wie individuell eine gewaltige Herausforderung. Das Denken in den Bahnen des erfolgreichen Modells der Nationalität mit ihrer Einsprachigkeit in der jeweiligen Hochsprache ist ganz tief im Bewusstsein verankert. Die europäische Union ist nun allerdings ein Raum, in dem dieses Denken herausgefordert wird, dass wir zu einem neuen Sprachdenken kommen, das einerseits nicht einfach zurückfällt in die Zeit vor diesem „Projekt Nation“, das aber andererseits in der Lage ist, ein Konzept zu entfalten, in dem Mehrsprachigkeit einen substanziell anderen Stellenwert hat, als das „Projekt Nation“ erlaubt. Das fängt dann damit an, dass wir eine andere Art von Demokratie brau- 
chen, um der auf dieser Tagung angesprochenen Faktizität in urbanen Räumen Europas gerecht zu werden; derzeit aber bleiben wir sozusagen gegenüber diesen Entwicklungen zurück.

Mein dritter Punkt: Sprache ist nicht einfach nur in einem kleinen Kreis selbstgenerierend, der im Übergang von einer Generation zur anderen die Sprache weiterträgt. Sprache ist eine zentrale Aufgabe der Bildungsinstitutionen. Das gehört mit zu dem Kontext des „Projekts Nation“ und das wird auch so bleiben. Unsere Berücksichtigung von Sprache in den Schulen ist aber ebenfalls ganz bezogen auf dieses Konzept herrschender europäischer Nationalsprachen. Da gibt es Deutsch, da gibt es Englisch, da gibt es Französisch und, wenn es gut geht, vielleicht noch ein paar andere Sprachen in den Schulen einzelner bundesdeutscher Länder. Wir brauchen aber als Ausdruck eines veränderten Sprachdenkens in Deutschland und ganz Europa ein Gesamtsprachencurriculum. Und dieses Gesamtsprachencurriculum wird auch in der Lage sein, die Rolle der tatsächlich gebrauchten und der Bildungssprachen gegenüber den Schülern zu verantworten in Mathematik das ist auf der Tagung von Rosemarie Tracy angesprochen worden - in vielen anderen Fächern. Das wirkt alles separiert nebeneinander her, geht in Umsetzung der jeweiligen Universitätsdisziplinen ... Ein Gesamtsprachencurriculum kann darüber hinaus wirken. In einem solchen Gesamtsprachencurriculum ist Sprache umfassend mit den verschiedenen Basisqualifikationen mit zu berücksichtigen. Das hat nicht zuletzt etwas mit der illusiologischen Dimension zu tun. Und ich denke, damit diese, damit ein neuer Stellenwert von Sprache, der innerhalb des schulischen Gesamtcurriculums Realität werden kann und anerkannt werden kann, bedarf es unter anderem einer anderen Qualifizierung der Lehrenden und der zukünftigen Lehrenden. Wir brauchen eine universitäre Ausbildung, in der Pflichtelemente in Bezug auf Sprache für alle zukünftigen Lehrerinnen und Lehrer vorgesehen sind, wie das jetzt im nordrhein-westfälischen Modell zum ersten Mal so verankert worden ist. Wir brauchen Sprachkonsolenten Quasthoff hat vorher diese Frage gestellt - wir brauchen Leute, die Spezialisten und Spezialistinnen sind mit einer linguistischen Qualifizierung in den Institutionen der Bildung sowohl in Bezug auf die Erzieherinnen und Erzieher wie in Bezug auf die Lehrerinnen und Lehrer. Hier ist Politik gefordert. Politik in einem sehr schwierigen Umfeld. Stichwort: Föderalismus der Bundesrepublik Deutschland.

Mein vierter Punkt: Kommunitäre Dimension. Individuell und gesellschaftlich stehen wir vor der Herausforderung einer multiplen Identitätsbildung und das ist kein leichtes Ding. Machen wir uns da keine Illusionen. Diese multiple Identitätsbildung setzt eine Art von praktizierter AlltagsMultikulturalität um, Umgang mit dem Fremden, ohne dass ich mich in meiner Identität gefährdet sehe, durch den Umstand dieser differenzierten Realität, die unsere sprachliche Wirklichkeit heute anstellt und hat. Das 
heißt, weggehen von dem, was Herr Androutsopoulos jetzt eben benannt hat, diesen Modellsatz der Defizite, hin zu einer neuen Sicht, in der der Ressourcencharakter dieser Mehrsprachigkeit, dieser faktischen Mehrsprachigkeit, als gesellschaftlicher Realität auch eine gesellschaftliche Anerkennung gewinnt.

Mein letzter Punkt: Das heißt nämlich für unsere Disziplin oder unsere Disziplinen, dass wir uns auch, und das wird auch ein sehr komplexer Prozess, denke ich, und ein sehr herausfordernder Prozess, dass wir uns auf den Weg machen müssen zu einer eingreifenden Linguistik. Wir sind darin vielfach, also die Disziplin ist in vielfältiger Weise ein gebranntes Kind. Die Reaktion ist dann, sozusagen, ein Rückzug auf eine deskriptive, deskriptiv orientierte Selbstverständigung. Ich denke, wir tun gut daran, wenn wir darüber hinausgehen, nicht alte Fehler wiederholen, sondern, sozusagen in einem solchen europäischen Mehrsprachigkeitshorizont, Sprache in einer neuen Weise mehr in die öffentliche Diskussion bringen.

[Moderation]: Damit wären wir in gewisser Weise die theoretisch wissenschaftlichen Positionen durchgegangen. Das Goethe-Institut und andere Institutionen haben es mit einer anderen Ebene zu tun, nämlich mit dem Sachverhalt, wie komplex dieser auch immer sein mag, gewisse Anforderungen im Hinblick auf mehrsprachiges Fungieren in einem dominant deutschsprachigen Kontext vorzubereiten und möglicherweise abzuprüfen. Die Frage, wie das und was da geschieht, ist eine praktische Probe aufs Exempel für das angemessene Umgehen mit den Bedingungen moderner gesellschaftlicher Mehrsprachigkeit - und nicht ohne politische Implikationen.

\subsection{Matthias Makowski (München)}

Als ich vor gut einem halben Jahr des Plakats an einer deutschen Universität ansichtig wurde, habe ich mir gedacht: „Aha! Das Deutsch der Migranten. Da könnte vielleicht das Goethe-Institut etwas dazu zu sagen haben." Warum? Das Thema „Migration“ ist eines der großen Leitthemen unserer kulturellen Programmarbeit im Ausland. Wir sind als Goethe-Institut, Sie wissen das wahrscheinlich, an hundertfünfzig Orten in der Welt vertreten, mit großen, mit kleinen Instituten und dort, wo wir in partnerschaftlichem Dialog versuchen, ein kleines Schaufenster Deutschlands zu sein, sind wir in ganz vielen Ländern an ganz vielen Standorten ganz intensiv und viel intensiver in Migrationsprozessen involviert, als wir es hier in Deutschland sind. Wenn wir eine Bewertung vornehmen würden, die haben wir hier jetzt noch nicht vollzogen, und das werden wir wahrscheinlich auch nicht tun, aber die Brisanz der Problematik ist an vielen Stellen der Erde weitaus größer als wir sie hier in Deutschland sehen. Also das ist der erste Ansatz, weshalb das 
Goethe-Institut alle Fragen spannend findet, die mit Migration zu tun haben. Wir mögen das Wort nicht, aus den verschiedenen Gründen. Wir versuchen auch, um politisch umfassender antworten zu können, das als ein Phänomen der Mobilität zu betrachten. Wir wissen, dass wir auch in gewissem Umfang eine klare Position vermeiden, wenn wir das Ganze mit dem schöneren Wort „Mobilität“ bezeichnen, aber letztlich gehört die Migration doch in diesen Gesamtkontext. Für das Goethe-Institut ist das Deutsch der Migranten das Deutsch, das sie „können“: Das Deutsch der Migranten wird geprüft. Es wird geprüft ganz im Sinne einer normgesetzkonsolidierten Einsprachigkeit. Geprüft wird es nur unter anderem durch das GoetheInstitut und diese Prüfungen haben, im Unterschied zu früher, allerdings heute Folgen. Als ich zum Goethe-Institut kam, gab es natürlich auch schon Prüfungen, Sprachprüfungen, die waren auch wichtig für uns, aber es hatte auch etwas von „l'art pour l'art“. Es hat natürlich auch etwas mit dem Schulzugang zu tun gehabt, DSH (Deutsche Sprachprüfung für den Hochschulzugang) und alles, was im Hintergrund steht, aber im Grunde: Existenzielle Folgen hatten solche Prüfungen in der Regel nicht. Das hat sich geändert. Es wird geprüft in den Herkunftsländern von Migranten, im Rahmen des Ehegattennachzugs. Das ist eine Geschichte, die mit dem Jahr 2007 begonnen hat, mit dem Gesetz, demzufolge eine Vielzahl von Menschen, die nach Deutschland zuwandern, vor der Einreise Grundkenntnisse der deutschen Sprache nachweisen müssen. Und dazu bedient man sich eines Tests, zum Beispiel „Sprachdeutsch 1“ des Goethe-Instituts. Das misst auf dem Niveau des gemeinsamen europäischen Referenzrahmens, das Niveau A1. Das macht nicht nur das Goethe-Institut, das machen auch Andere.

Wir messen und wir prüfen die deutsche Sprache aber auch an einer anderen Stelle. Man prüft auch in Deutschland die deutsche Sprache. Der Deutschtest für Zuwanderer, den das Goethe-Institut im Auftrag des Bundesministeriums des Innern (BMI) entwickelt hat und den es seit 2009 gibt, ist ein für die Zielgruppe der in Deutschland lebenden Menschen nichtdeutscher Muttersprache ausgerichteter Test, der das Niveau B2 prüft. Das ist nicht ganz so problematisch, weil damit keine Abschiebung verbunden ist für den Fall, dass man ihn nicht besteht. In anderen Ländern ist das bedauerlicherweise durchaus eine reale Frage.

Doch wenn man ein Kulturinstitut ist, wenn man sich auch anschaut, aus welchem Kontext die Mitarbeiter des Goethe-Instituts kommen, kann man sich vorstellen, dass diese plötzliche politische Dimension unseres Handelns, ein Institut, das immer auch auf seine politische Unabhängigkeit besonderen Wert gelegt hat, zu heftigen internen Diskussionen geführt hat. Ein wichtiges Argument dabei war aber auf jeden Fall, dass es darum ging, die unabweislichen gesetzlich geforderten Schritte möglichst professionell umzusetzen. Gerechtfertigt haben wir diesen Zugang damit, dass wir um die Prüfung herum einen Cordon sanitaire gelegt haben, mit vielen Beratungs- 
angeboten, Hilfsangeboten, bis hin zu psychologischer Beratung und der Beschäftigung mit Aufgaben, mit denen wir noch nie zu tun hatten. Beispielsweise haben wir in diesem Kontext mit vielen Menschen zu tun, die das Schriftsystem erst erwerben müssen.

Ich will zum Ende kommen. Ich möchte nur deutlich machen: Das Thema, das wir vorhin hier so reflexiv diskutierten, hat beim Goethe-Institut eine richtige Krise ausgelöst, die dazu führte, dass wir uns bekennen mussten, wollen wir da mitmachen, oder wollen wir da nicht mitmachen. Und da man sich nicht waschen kann ohne sich nass zu machen, haben wir uns dann entschieden, wir machen da mit und wissen, dass wir uns mit diesem Projekt durchaus anfechtbar machen. Und vielleicht kann ich an dieser Stelle erst einmal aufhören.

\section{Die Diskussion auf dem Podium}

Drei Punkte spielten in der weiteren Diskussion auf dem Podium die zentrale Rolle; es ging um das Konzept „Deutsch der Migranten“, um die Frage der gesellschaftlichen „Regulierung“ der Diversität und um die Frage eines demokratisch angemessenen Konzepts von Integration - und einer entsprechenden Praxis.

Konrad Ehlich betonte dabei insbesondere den Zusammenhang der politischen Bedeutung europäischer Konzepte von Mehrsprachigkeit mit einem neuen Verständnis von Integration in der primär monolingual orientierten Gesellschaft der Bundesrepublik. In dieser Hinsicht sei der bewusste Schritt hin zu den Integrationskursen, die nicht einfach auf sprachliche Assimilation zielten, ein merklicher Fortschritt. Im selben Sinne erläuterte Matthias Makowski das Konzept des Goethe-Instituts, den Erwerb von Deutsch als Fremdsprache als Teil der Förderung einer europäischen Mehrsprachigkeit zu fördern, als eine Art Skandalon für die Sprecher vergleichsweise großer Sprachen wie des Deutschen, aber als eine Art nützlicher Irritation, die darauf aufmerksam macht, dass Mehrsprachigkeit eigentlich eine Leistung ist, die eher von den Sprechern ,kleiner" Sprachen erbracht wird, die aber auch von den Sprechern der ,großen“ und Schulfremdsprachen zu erwarten wäre. Frau Franceschini hob in diesem Zusammenhang besonders hervor, dass es nicht um ein einfaches Mehr geht, sondern dass eine funktionale Mehrsprachigkeit anzustreben ist, die den differenzierten sprachlichen Bedürfnissen des Individuums in einer Gesellschaft entspricht. Bei der Begegnung mit unterschiedlichen sprachlichen Profilen geht es darum, Diversität als selbstverständlich zu akzeptieren, und die Existenz einer multiplen Identität nicht als Bedrohung anzusehen. Von einer anderen Seite griff Jannis Androutsopoulos den Gedanken der Diversität auf, nämlich in einer Aufforderung an die Politik, sich an der „kundenorientierten“ Differenzierungsfähigkeit der Wirtschaft und der Werbung ein Beispiel zu nehmen. 
Einvernehmlich wurde beklagt, dass offenbar der Aufbruch zu einem Mehrsprachigkeitskonzept („Muttersprache +2 ") in der europäischen Union im politischen Geschäft wieder ermattet sei.

Nach der Öffnung in das Publikum wurden weithin diese Punkte weiter diskutiert. Zum Abschluss kam es zu einer Diskussion über eine sinnvolle Interpretation der mit dem Tagungstitel „Das Deutsch der Migranten“ angesprochenen Sachverhalte. Man konnte sich vergleichsweise leicht darauf verständigen, dass die Verhältnisse von Mehrheitssprache, Sprachkontakt und Mehrsprachigkeit, die sich in den letzten Jahrzehnten in Folge von Migration ergeben haben, im Hinblick auf die sprachlichen Anforderungen der Beteiligung des Einzelnen an der Interaktion in der Öffentlichkeit eines demokratischen deutschsprachigen Staates sowohl einer wissenschaftlichen Untersuchung wie einer Diskussion über die daraus sich ergebenden Befunde zu ziehenden praktischen Folgen wert sind. Und das ist nur einer der Texte, zu denen der kurze Titel passt. 